522 Fresenius: Veber die richtige Ausführung und die Empfindlichkeit

stehenden Mitteln auf dem Wege des Versuchs zur Klärung wichtiger Fragen der Feuerungskunde beizutragen and ich erfreue mich lierbei, wie ich dankbar anerkenne, der lebhaften Unterstützung von Männern der Wissenschaft wie der Praxis.

Den Angriffen des Herrn Wag a e r gegenẗber habe ich mein letztes Wort gesprochen.

\title{
Mittheilungen ans dem chemischen Laboratorium des Prof. Dr. R. Fresenius zu Wiesbaden.
}

Ueber die richtige Ausführung und die Empfindlichkeit der . Fresenius-Babo'schen Methode zur Nachweisung des Arsens.

\section{Von \\ W. Fresenius.}

Die directe Veranlassung zu der nachstehenden Arbeit, welche ich auf Anregung meines Vaters unternahm, war die Beschreibung des Fresenius-B abo'schen Verfahrens in der kürzlich erschienenen dritten Lieferung von Graham-Ottos Lehrbuch der anorganischen Chemie.*)

Dieselbe weicht nämlich in verschiedener Hinsicht sehr erheblich von den Angaben ab, welche sich in der ursprünglichen Abhandlung**) oder auch in der Anleitung zur qualitativen Analyse von R. Fresenius finden, und zwar betrefien die Aenderungen zum Theil gerade solche Punkte, auf welche im Original ein ganz besonderer Werth gelegt wird, die ganz speciell zum Wesen der Methode gehören. Da man aber sofort erkennt, dass diese Modificationen nicht alle auf einmal und namentlich nicht erst jetzt bei der Bearbeitung der nevesten Auflage des Graham-Otto'schen Lehrbuchs angebracht worden sind, so verglich ich die Beschreibungen der Methode in sämmtlichen mir zugänglichen neueren Werken, um zu erfahren, von wem und aus welchem Grunde die Abänderungen vorgeschlagen worden sind. ch kam d́abei zu dem merkwürdigen Resultate, dass ausser in ders Ischon erwähnten Anleitung zur qualitativen Analyse von R. Freseni u

*) Fünfte Auflage, bearbeitet von Dr. A. Michaelis.

**) Annalen d. Chemie u. Pharmacie 49, 287. 
nur noch in der Anleitung zur chemischen Analyse von H. Will and in den Lehrbüchern der anorganischen Chemie ron Gorup-Besanez und $\mathrm{Ph}$. Th. Büchner die richtige ursprüngliche Ausführungsweise angegeben ist, dass sich dagegen in allen anderen Werken, die uiberhaupt näher auf die Methode eingehen, melr oder weniger falsche Darstellungen finden.*)

Es scheint mir dies daher zu kommen, dass diese Bearbeitungen nicht direct nach dem Original gemacht sind, sondern dass in allen Fällen eine oder einige wenige Quellen benutzt wurden, in welchen sich schon die unrichtige Beschreibung fand; denn es kann doch unmöglich angenommen werden, dass sämmtliche Autoren selbstständig und in der nämlichen Weise eine Operation abgeändert haben, auf die in der ursprünglichen Ablandlung ganz besonders hingewiesen wird, zu deren Begründung eine eigene Versuchsreihe ausgeführt und genau beschrieben ist.

Als derartige gemeinsame Quelle scheint nun hauptsächlich Graham-Otto's Lehrbuch der anorganischen Chemie, resp. Otto's Anleitung zur Ausmittelung der Gifte gedient zu haben, wenigstens erscheinen alle Abänderungen, um welche es sich hier handelt, in den verschiedenen Auflagen dieser beiden Werke als selbstständige Vorschläge ; anfangs neben der ursprünglichen Form als Vereinfachungen oder Verbesserungen, damn - in späteren Bearbeitungen - allein, oder doch nur unter kurzer Erwähnung des alten Verfahrens. Und mit der Umgestaltung der Methode selbst ändert sich auch die Beurtheilung derselben; je mehr die alte Form des Verfahrens zurücktritt, um so ungünstiger wird es auch besprochen, so dass es schliesslich in der dritten Auflage der Ausmittelung der Gifte**) bei der Vergleichung der Methode mit der von Berzelius-Marsh (Seite 64) heisst, dass diese (die Marsh'sche) sich durch ihre Einfachheit und Leichtausführbarlieit auszeichne und dass dabei das Resultat weit weniger von der Umsicht und

*) Unter anderen Werken, z. B. Rose, Handbuch der analytischen Chemie, in den älteren deutschen und der französischen Ausgabe. Graham0 tto, Lehrbuch der anorganischen Chemie. Otto, Anleitung zur Ausmittelung der Gifte. Handwörterbuch der Chemie, in beiden Ausgaben. Mohr, Toxikologie. Dragendorff, gerichtlich-chemische Ermittelung von Giften.

**) Es muss diese Bearbeitung, obgleich der Zeit nach früher als die Darstellung in dêr vierten Auflage von Graham-Otto's Lehrbuch, doch als eine weitergehende und in diesem Sinne spätere bezeichnet werden, da hier sowohl die ältere Form des Verfahrens ganz weggelassen, als auch das Urtheil, wenn auch nur in Kleinigkeiten, schärfer ist. 
524 Fresenius: Ueber die richtige Ausführung und die Empfindlichkeit

Vorsicht des Arbeitenden abhängig sei, wie bei dem Verfahren von Fresenius und Babo. Hiernach erscheint dieses letztere zum Mindesten als ganz überfüssig, welche Ansicht noch viel unzweideutiger auf Seite 87 ausgesprochen ist, wo es heisst:

$\gg$ Dieser Vorzug des Verfahrens hat jetzt die Bedeutung verloren, »das Verfahren kann daher aufgegeben werden, da es, wie schon oben »gesagt, dem Verfahren Berzelius-Marsh an Empfindlichkeit nach»steht. Schneider, ein geübter Arbeiter, konnte in meinem Labo»ratorio aus einem Milligramm arseniger Säure keinen Spiegel erhalten.«

Die letzten Zeilen stellen die Methode nicht nur als überflüssig, sondern auch als ungenau and deshalb auch als unzweckmässig, als überwunden dar.

Diesem Urtheil stehen nun zunächst die Angaben von Fresenius und $\mathrm{Babo}$ in der ursprünglichen Abhandlung gegenüber, in welcher dieselben das Verfahren als ein »unter allen Umständen sicheres « bezeichnen und in welcher sie auf Seite 304 angeben, dass man nach demselben $\gg$ aus $1 / 4 m g$ Schwefelarsen and selbst aus noch kleineren Quantitäten noch vollkommen deutliche Spiegel erhält und zwar mit vollkommenster Sicherheit und ohne irgend eine Ausnahme «. Ebenso hat sich auch die Methode bei vielfacher Anwendung im hiesigen Laboratorium stets bew ährt. *)

Wenn man sich bei diesem directen Widerspruch der Urtheile klar macht, dass das eine sich auf die ursprüngliche, das andere auf die modificirte Form der Methode bezieht, so liegt es sehr nahe, den Grund dieses Widerspruches eben in den erwähnten Abänderungen zu suchen. In diesem Falle würden dieselben - mindestens zum Theil - statt Verbesserungen gradezu. Verschlechterungen der Methode sein und es würde die Gefahr drohen, dass die an sich ganz gute Methode, die jedoch schon jetzt hauptsächlich in der modificirten Gestalt bekannt ist, nach und nach durch diese sogenannten Vereinfachungen völlig in Misscredit gerathen würde.

Es erschien mir daher geboten, die verschiedenen Abänderungsvorschläge einmal auf ihre Nothwendigkeit oder auch nur Zulässigkeit zu prüfen, zu untersuchen, welchen Einfluss sie anf die Genauigkeit des Resultates haben und bei dieser Gelegenheit auch die Empfindlichkeit

*) Vergl. R. Fresenius Anleitung zur qualitativen Analyse, 14. Auflage S. 380 , Anmerkung. 
der Methode in ihrer alten Form durch etwas ausführlichere Experimente festzustellen.

Bei der Besprechung dieser Untersuchungen, zu der ich jetzt übergehe, setze ich das Princip der ursprünglichen Methode - Reduction von Schwefelarsen durch Erhitzen mit einer Mischung ron Soda und Cyankalium im Kohlensäurestrom und Erzeugung eines Spiegels von metallschen Arsen als bekannt voraus und will nur auf die einzelnen Abänderùgsvorschläge und zwar in der Reihenfolge, wie sie der Zeit nach gemacht worden sind, specieller eingehen. Es handelt sich dabei im Wesentlichen um drei Punkte: 1) um die Art der Erhitzung, 2) um die Verbindungsform des Arsens, welche zur Reduction benutzt wird und 3) um die Gasatmosphäre, in welcher die Reduction rorgenommen wird.

Die einzige sonstige Abänderung, nämlich die Ersetzung des ursprünglichen einfachen Entwicklungskolbens durch einel constanten Grasentwicklungsapparat, brauche ich nicht näher zu besprechen, da es offenbar ein grosser Vortheil ist, dass man durch dieselbe in den Stand gesetzt wird, dem Gasstrom ganz genau die gewünschte Stärke zn geben, während man fruher eben einfach so lange warten masste, bis die Entwickinng sich hinreichend verlangsamt hatte. Zwar hatte man dann auch sicher die Gewissheit, dass der Apparat ganz mit Kohlensäure gefült war, aber es darf doch wohl angenommen werden, dass auch jetzt jeder, der die Methode anwendet, diesen Zeitpunkt abwartet, ehe er mit dem Erhitzen beginnt. Der Grund, warum nicht von vornherein ein continuirlicher Gasentwicklungsapparat angegeben wurde, ist nur der, dass es damals viel schwieriger war einen derartigen Apparat zu erhalten, and es ist deshalb um so wunderbarer, dass sich grade diese wirkliche Verbesserung so langsam verbreitet hat, dass erst in der allerneuesten Zeit die alte Abbildung aus den Büchern verschwunden ist.

\section{1) Die Art der Erhitzung.}

In der Originalabhandlung von R. Fresenius und L. T. Babo sind anf Seite 305 einige Versuche angefüht, durch welche diese Frage eigentlich schon an und fur sich entschieden ist, ich will deshalb wegen der Wichtigkeit derselben ihre Beschroibung wörtlich folgen lassen:

* Erhitzt man metallisches Arsen in einer langen Glasrohne und »leitet einen langsamen Strom von kohlensaurem Gas darüber, so erhält $\gg$ man hinter der erhitzten Stelle einen Metalspiegel, wahrend gleichzeitig »aus dem offenen Ende der Röhre eine bedeutende Menge Arsen in 
$\gg$ Dämpfen entweicht und die Luft mit Knoblauchgeruch erfüllt. Erhitzt »man die Röhre noch an einer weiter nach vorn gelegenen Stelle, so »wird man finden, dass sich hinter derselben ebenfalls ein Metallspiegel »absetzt, und erhitzt man stark und ist der Strom des Gases langsam, »so gelingt es leicht, auf diese Weise beinahe alles Arsen in der Röhre $\gg$ metallisch niederzuschlagen. Man verdichtet demnach Arsendampf, »oder in dem Gas suspendirte Arsentheilchen, denn in einer dieser »beiden Formen ist ja das Arsen jedenfalls in der Röhre enthalten, $\rightarrow$ durch Erhitzen.

$\gg$ Diese Thatsache, welche beim ersten Anblick unwahrscheinlich $\gg$ dünkt, findet in Folgendem ihre Erklärung. Bei dem Erhitzen ver$\gg$ dampft Arsen; der durch die Hitze expandirte Dampf kommt mit der $\gg$ kalten Glasröhre in Berührung, ein Theil desselben schlägt sich an »dieselbe nieder, ein anderer Theil kühlt sich in dem Gasstrom ab und $\gg$ die verdichteten Arsentheilchen werden, in demselben suspendirt, fort$\gg$ geführt, behalten aber immer noch diejenige Temperatur, welche eine $\gg$ Oxydation derselben an der Luft bedingt, daher der Knoblauchgeruch »beim Austreten des Gases aus der Röhre. Erhitzt man aber einen » Theil der Röhre, durch welche das mit Arsentheilchen beladene Gas »streicht, so werden dieselben an dieser. Stelle wiederum in expandirten »Arsendampf verwandelt und die obige Erscheinung, die Entstehung $\gg$ eines Metallspiegels, muss demnach natürlicherweise wiederum eintreten.

$\gg$ Auf dieser und auf keiner anderen Ursache beruhen auch die »Erscheinungen, welche man beim Erhitzen ron metallischem Arsen oder » von einem Gemenge von Schwefelarsen mit Soda in Wasserstoffgas $\gg$ beobachtet hat.»

Aus den Verhältnissen, die durch diese Versuche klar gelegt. sind, ergibt es sich nun ganz von selbst, dass man $n i c h t$ (wỉe es in fast allen Büchern angegeben ist) $\mathrm{n} \mathrm{ur}$ die Stelle der Röhre, wo das Gemisch der Arsenverbindung mit Soda und Cyankalium liegt, erhitzen darf — weil dann ja die Hauptmenge des Arsens nicht als Spiegel erhalten wird sondern, dass man, wie dies von R. Fresenius und L. v. Babo vorgeschrieben wird, an zwei Stellen, also mit zwei Lampen erhitzen muss. Und zwar erhitzt man, nachdem die Röhre ganz ausgetrocknet ist, zuerst die Stelle der Röhre, wo die Verengerung anfängt, zum Glühen und beginnt erst, nachdem dies erreicht ist, mit der Erhitzung des Gemenges, welche, wie schon gesagt, mit einer zweiten Lampe auszuführen ist. Wenn man nun aber auch die obigen'Versuche gar 
nicht kennt, so sieht man doch sofort, dass dieses an sich ja immerhin merkwürdige Erhitzen mit zwei Lampen nicht o hne Grund im Original angegeben ist. Wenn man daher die eine Lampe der Einfachheit halber wegfallen lassen wollte, so musste man doch erst beweisen, dass dadurch auch die Genauigkeit der Methode nicht beeinträchtigt werde.

Es ist ein solcher Beweis jedoch nie versucht worden, sondern das Erhitzen mit einer Lampe wird in der dritten Auflage von Gra ham 0 tto's Lehrbuch einfach in der Weise vorgeschlagen, dass es, nachdem zuerst die ursprüngliche, richtige Vorschrift angeführt ist, weiter heisst:

»Weil das Erhitzen der Reductionsröhre mit zwei einfachen Spiritus»lampen lästig ist, indem leicht eine Lampe der anderen im Wege steht » und weil jeder Hauch die Flanme von der Röhre wegtreibt, so kann $\gg$ man auch zum Erhitzen eine Argand'sche Spirituslampe anwenden. »Man bringt dann das zu reducirende Gernenge auf angegebene Weise »weiter in die Röhre hinein, nahe der Stelle, wo sich die Röhre zur »Verengerung wölbt, stellt unter dieselbe die brennende Spirituslampe $\gg$ mit nur wenig herausgeschraubtem Dochte, trocknet zuvörderst das $\gg$ Gemenge in dem heissen trockeuen Luftstrome auf das Sorgfältigste »aus und erhitzt schliesslich das Gemenge zum Schmelzen. Das redu»cirte und verfiüchtigte Arsen setzt sich nahe der Wölbung der Röhrè $\gg$ oder in der Wölbung als glänzender Spiegel an, leicht aber entsteht $\gg$ dann auch ein Anflug in der Röhre vor dem Gemenge.

Man erçennt, dass bei der Anwendung e in er Argand'schen Weingeistlampe, ${ }^{*}$ ) wegen der breiten Flamme derselben und bei dem Vorschieben des Gemenges bis nahe an die Wölbung der Fehler, welcher durch das Weglassen der zweiten Lampe entsteht, wesentlich reducirt werden kann, weil, wenn man zuerst nur die WöLbung zum Glühen erhitzt und dann mit der Lampe allmählich nach dem Gasentwicklungsapparate hinrückt, die Arsendämpfe stets durch eine glühende Stelle der Röhre hinstreichen und dann einen Spiegel in dem verengten' Theile erzeugen können. Wenn man dabei den Gasstrom sehr langsam macht, so ist die Menge des Arsens, die aus der Spitze der Röhre entweicht, verhältuissmässig klein; man erhält aber in diesem Falle (auch beim Erhitzen mit zwei Lampen) leicht »einen Anflug in

*) Die Argand'sche Lampe gestattet wegen ihrer Grösse nicht wohl ein Erhitzen der Röhre ihrer ganzen Länge nach und erschwert dadurch das völlige Austrocknen ganz erheblich.. 
528 Fresenius: Veber die riehtige Ausführung und die Empfindlichkeit

der Röhre vor dem Gemenge «, wie dies Otto an der citirten Stelle angibt.

Um mit diesem modificirten Verfahren keine ganz schlechten Resultate zu erlangen, muss man also die genannten Maassregeln und zwar mit $\gg$ grosser Vorsicht und Umsicht « anwenden, während demgegenüber die ursprüngliche Methode als einfach und leicht ausführbar zu bezeichnen ist.

$J$ ulius 0 t to hat nun allerdings diese Punkte, welche den Fehler auf das möglichst kleinste Maass beschränken, stets eingehalten, denn auch in der dritten Auflage der Ausmittelung. der Gifte, in der das Erhitzen mit zwei Lampen gar nicht mehr erwähnt ist, findet sich die Argand'sche Weingeistlampe und die Lage des Reductionsgemisches nahe an der Wölbung vorgeschrieben. Aber in die anderen Werke gingen diese Vorsichtsmaassregeln nicht über, zum Erhitzen wird 'einfach ein Bunsen'scher Brenner*) vorgeschlagen und die Lage der zu erhitzenden Substanz ist in der fünften Auflage von O'to's Ausmittelung der Gifte als »in der Mitte des cylindrischen Theiles der Röhre « angegeben. Ebenso muss man die Angaben mehrerer anderer Werke**) auffassen, welche, obgleich sie nur e in e Lampe erwähnen, die Abbildung der beschickten Röhre enthalten, wie sie sich in R. Fresenius Anleitung zur qualitativen Analyse oder auch in den früheren Auflagen von GrahamOtto's Lehrbach findet, und die sich auf das Erhitzen mit zwei Lampen bezieht, denn es liegt bei derselben die Substanz ziemlich in der Mitte der Röhre und es ist noch durch einen besonderen Buchstaben die Stelle bezeichnet, welche (eigentlich) durch die andere Lampe zum Glühen erhitzt werden soll. Erhitzt man aber das in der Mitte der Röhre liegende Gemisch nur mit einer Lampe, so muss sich nach den obigen Versuchen ein sehr grosser Theil des Arsens ganz verflüchtigen and wenn sich überhaupt ein' Spiegel bildet, so wird er nicht in der Verengerung, sondern irgendwo im weiten Theile der Röhre entstehen und dadurch, wenn er klèin ist, viel leichter übersehen werden. ${ }^{* * * *}$ )

*) Derartige Abbildungen finden sich z. B. in Mohr's Toxikologie und in Scherer's Lehrbuch der Chemie.

**) Z. B. Handwörterbuch der Chemie, oder Wilke, Anleitung zur chemischen Analyse.

***) Das Extrem von falscher Darstellung der Erhitzungsweise findet sich in Rose's Handbuch der analytischen Chemie (ältere deutsche oder anch französische Ausgabe). Es wird dort nämlich nicht blos n ur eine L a mpe angegeben, 
Um nun die Thatsachen, welche sich aus dem bisher Gesagten ergeben, auch noch experimentell zu beweisen, stellte ich folgende Versuche an, die zugleich die Empfindlichkeit der Methode, bei richtiger Ausführung, erkennen lassen.

Durch Auflösen von arseniger Säure in Wasser mit Hülfe von kohlensaurem Natron stellte ich mir eine Flüssigkeit her, welche in einem Cubikcentimeter ein Milligramm arseniger Säure und zehn Milligramm wasserfreies kohlensaures Natron enthielt. Von dieser Flüssigkeit maass ich nun mit einer Quetschhahnbürette verschiedene mal je einen Cubilicentimeter $a b$ und dampfte denselben in einem Porzellanschälchen auf dem Wasserbade zur Trockne, kratzte den Rückstand mit einem Platinspatel los und mischte denselben durch Zusammenreiben in einer kleinen Achatreibschale mit etwa der zehnfachen Menge eines Gemenges. von einem Theil reinem Cyankalium und drei Theilen wasserfreiem kohlensaurem Natron.*) Dann brachte ich unter Vermeidung jedes Verlustes die Mischung in eine Reductionsröhre, trocknete im Kohlensäurestrom durch gelindes Erhitzen der ganzen Röhre sehr sorgfältig aus und erhitzte dann einerseits nach der eigentlichen Vorschrift mit zwei Lampen, andererseits, um eine breite Flamme zu haben, mit einer Maste'schen Gaslampe (wobei die Mischung bis nahe an die Wölbung vorgebracht wurde) und endlich auch uur mit einer Bunsen'schen Lampe (das Gemenge lag in der Mitte des cylindrischen Theiles der Röhre).

Ich erhielt dabei in allen Fällen starke Spiegel, von denen aber die mit zwei Lampen erzeugten bei weitem grösser und namentlich auch besser in dem verengten Theile angelegt waren, auch mit der Maste'schen Lampe erhielt ich sie noch relativ sehr schön; beim Erhitzen mit einer Bunsen'schen Lampe entstand zwar auch stets ein ganz unverkennbarer Spiegel, derselbe war aber von ganz unregelmässiger Form und trat, je nach den Verhältnissen, an irgend einer Stelle im weiten Theile des Rohres auf.

sondern die Abbildung zeigt anch ein Reductionsrohr, welches überhaupt keine Verengerung hat. Diese Rose'sche Beschreibung ist übrigens jedenfalls selbstständig und nicht aus Graham-0 tto geschöpft.

*) Dass ich mich durch bosondere Vexsuche davon überzeugte, dass meine Reagentien absolut arsenfrei waren, bedarf wohl kaum der Erwähnung. Ich will jedoch hier nochmals darauf aufmerksam machen, dass man diese Prüfung nie unterlassen darf, indem sowohl das kohlensaure Natron als auch das Cyankalium vielfach einen, wenn auch nur spurenhaften, Arsengehalt zeigen. Vergl. die Angaben von R. Fresenius in dieser Zeitschrift 6, 201. 
530 Fresenius: Ueber die richtige Ausführung und die Empfindlichkeit

Ich kann dem gegenüber die Schneider'schen Versuche, die Otto (a. a. 0.) mittheilt, nicht für richtig halten. Entweder hat Schneider nicht wirklich ein Milligramm arsenige Säure angewandt resp. dasselbe nicht wirklich völlig in die Reductionsröbre gebracht, oder er hat die Röhre nicht wirklich mit Kohlensäure gefüllt, *) so dass das Arsen sich sofort wieder oxydirte, oder er muss durch einen unverhältnissmässig starken Strom von Kohlensäure den Arsendampf aus der Röhre hinausgetrieben haben, ohne dass derselbe Zeit gehabt hätte, einen. Spiegel zu erzengen.

Um das Verhalten von noch kleineren Arsenmengen zu untersuchen, stellte ich mir durch Verdünnen Lösungen dar, die nur $1 / 10$ resp. ${ }^{1 / 100} m g$ arsenige Säure in $1 c c$ enthielten. Ich dampfte von denselben je einen oder mehrere Cubikcentimeter zur Trockne, fügte aber jedesmal vorher, um keinen zu kleinen Rückstand zu erhalten, $1 c c$ einer 1 procentigen lösung von kohlensaurem Natron zu. Die feste Masse behandelte ich ganz wie oben angegeben. Ich erhielt dabei, wenn ich nur mit e in er Lampe erhitzte, zwar auch noch bei $1 / 10 m g$ arseniger Säure einen kleinen Spiegel, ob man aber jedesmal einen solchen erhalten wird, erscheint mir zweifelhaft; noch-kleinere Arsenmengen dürften beim Erhitzen mit einer Lampe wohl nur ausnahmsweise einen erkennbaren Spiegel liefern. Beim Exhitzen mit $z$ wei Lampen dagegen fand ich eine sehr viel grössere Empfindlichkeit wie man aus folgender Zasammenstellung meiner Resultate ersehen wird.

1 mg arseniger Säure gab in dem engeren Theile ${ }^{* *}$ ) der Reductionsröhre einen starken Spiegel. Es war nicht möglich zu vermeiden, dass während des Versuches, also während die Röhre glühte, ein Theil des Arsens entwich und die Luft mit Knoblauchgeruch erfüllte.

$1 / 10 \mathrm{mg}$ arseniger Säure gab einen schwächeren, aber noch sehr schönen Spiegel in dem verengten Theile der Röhre, bei nicht zu raschem Gasstrome trat kein Knoblauchgeruch auf.

$1 / 20 \mathrm{mg}$ gab einen dem vorigen ganz ähnlichen nur entsprechend schwächeren Spiegel.

$1 / 50$ mg gab einen sehr dentlichen, braunen Anflug.

*) Wenn z. B. die Waschflasehe nicht völlig schliesst, so kann bei einem scheinbaren schwachen Gasstrom doch, auch nach längerer Zeit, in der Reductionsröhre noch so viel Luft sein, dass alles Arsen oxydirt wird.

**) Da die Wölbung selbst noch zu nahe der glühenden Stelle war, so konnte sich dạrin kein Spiegel absetzen. 
1/100 mg gab einen noch ganz deutlich erkennbaren, braunen Anflug, der - namentlich wenn man die Röhre auf weisses Papier legte - sehr schön hervortrat.

In den Fig. $26-28$ ist es versucht, die Spiegel von $1 \mathrm{mg}, 1 / 10 \mathrm{mg}$ und $1 /{ }_{100}^{\circ} m g$ arseniger Säure darzustellen.

Vergleicht man diese Abbildungen mit denen der Spjegel, die J. Otto nach dem Berzelius-Marsh'schen Verfahren erhielt, so Fig. 26,27 u. 28. wird man erkennen,

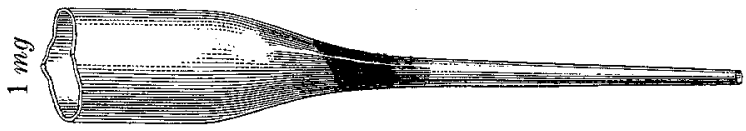
dass von einer erheblichen Differenz der Empfindlichkeit beider Methoden gar

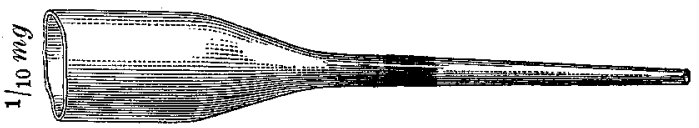
keine Rede sein kann, noch unzweifelhafter aber ergibt sich dies aus der Beschreibung,

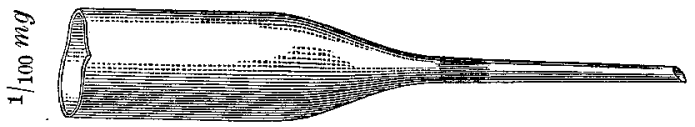
die $O$ tt $o$ von seinen Spiegeln gibt, denn dieselbe stimmt fast wörtlich überein mit

der oben gegebenen Darlegung meiner Resultate.

Es ist somit die Methode bei richtiger Ausführung vollkommen geeignet auch die kleinsten Arsenmengen mit völliger Sicherheit zu erkennen, ja die Empfindlichkeit*) derselben geht bedeutend über die Grenze des praktischen Bedürfnisses hinaus.

2) Die Verbindungsorm des Arsens, welche zur Reduction benutzt wird.

R. Fresenius und L. v. Babo bedienten sich des reinen Dreifach-Schwefelarsens, um daraus die Metallspiegel herzustellen, und sie erhielten damit sehr befriedigende Resultate. Gegen die Verwendung des Schwefelarsens ist jedoch späterhin der Einwurf erhoben

*) Ich will nicht versïumen, darauf hinzuweisen, dass nach den Angaben von J. Franck (vergl. diese Zeitschrift 5, 201) die Empfindlichkeitsgrenze der Marsh'schen Methode bei 1/3000 mg arseniger Säure, die des Fresenius-Babo'schen Verfahrens bei $1 / 500 \mathrm{mg}$ Schwefelarsen liegen soll. So kleine Mengen dürften sich jedoch wohl nur bei grösster Uebung und Vorsicht und selbst dann nicht jedesmal mit sicherheit auffinden lassen. 
532 Fresenius: Ueber die richtige Ausführung und die Empfindlichkeit

worden, dass dabei nicht alles Arsen in Dampf verwandelt werde, und dadurch die Empfindlichkeit bedeutend geringer sein müsse, als bei der Reduction einer Sauerstoffverbindung des Arsens.

Es gründet sich dieser Einwand auf Versuche von H. Rose*), durch welche derselbe nachgewiesen hat, dass beim Zusammenschmelzen von Schwefelarsen und Cyankalium sich ein Sulfosalz des Arsens bildet, welches durch das Cyankalium nicht angegriffen wird. Man erhält die in dem Rückstande bleibende Arsenmenge beim Aufösen desselben in verdünnter Salzsäure als gelbes Schwefelarsen. Ist der zu schmelzenden Masse eine gewisse Quantität freier Schwefel beigemengt, so entsteht überhaupt kein Arsenspiegel.

J. 0 t to hat, um den Verlust an Arsen zu vermeiden, vorgeschlagen, dass man das Schwefelarsen durch Erhitzen mit Salpetersäure in Arsensäure überführen, die Salpetersäure dann durch Eindampfen unter wiederholtem Befeuchten mit Wasser entfernen und den so erhaltenen Rückstand wie gewöhnlich reduciren soll. Man umgeht so allerdings den genannten. Uebelstand, allein das Verfahren hat das äusserst Missliche, dass, wenn nur noch eine Spur von Salpetersäure oder von einem Nitrate zurückbleibt, beim Erhitzen mit Cyankalium leicht eine Explosion eintreten kann. Yan muss deshalb mit grosser Vorsicht arbeiten und vor allem die Anwesenheit einer Basis vermeiden, welche zur Bildung eiries salpetersauren Salzes Anlass geben könnte.

Um mich zu überzeugen, ob der Fehler, der nach den Rose'schen Untersuchungen beim Reduciren von Schwefelarsen begangen wird, wirklich so gross ist, dass deshalb der 0 t to'sche Vorschlag trotz seiner Unannehmlichkeiten in der Ausführung als nothwendig angenommen werden müsste, stellte ich folgende Versuche an.

Ich fällte aus $500 c c$ der oben angeführten concentrirtesten Lösung von arseniger Säure nach dem Ansäuern mit Salzsäure das Arsen durch Einleiten von Schwefelwasserstoff aus. Den Niederschlàg von DreifachSchwefelarsen löste ich nach dem Auswaschen in Ammoniak und verdünnte diese Lösung auf $500 \mathrm{cc}$. Ich erhielt dadurch eine Flüssigkeit, die in einem Cubikcentimeter eine Menge von Schwefelarsen enthielt, welche $1 \mathrm{mg}$ arseniger Säure entspricht.

Aus dieser Lösung stellte ich mir durch Verdünnen eine zehnmal and eine handertmal schwächere dar. Ich verdampfte von diesen Lö-

*) Berichte der Akad. der Wissenschaften zu Berlin 1853, p. 441. 
sungen, wie oben, abgemessene Mengen unter Zusatz von je einem Cubikcentimeter der 1 procentigen Lösung von kohlensaurem Natron und verfuhr mit den Abdampfungsrückständen ganz wie oben angegeben.

Ich erhielt dabei Spiegel resp. Anflüge, welche mit den entsprechenden aus arseniger Säure dargestellten absolut gleich stark waren. Eine irgend merkliche Abnahme der Empfindlichkeit tritt also nicht ein, wenn man Dreifach-Schwefelarsen statt einer Sauerstoffverbindung anwendet, wenigstens dann nicht, wenn man eine genügende Menge von kohlensaurem Natron und Cyankalium anwendet, und wenn die ammoniakalische Lösung mit kohlensaurem Natron eingedampft wird, wie dies auch von R. Fresenius*) für den Fall empfohlen wird, dass man nur kleine Mengen von Schwefelarsen hat.

Der Rückstand von der Reduction der $1 \mathrm{mg}$ arseniger Säure entsprechenden Menge von Schwefelarsen gab, als ich ihn in verdünnter Salzsäure löste, keinen erkenmbaren Rückstand von Schwefelarsen und auch bei längerem Einleiten von Schwefelwasserstoff in diese Lösung schied sich kein Schwefelarsen aus. In anderen Fällen ist von R. Fresenius bei Anwendung von grösseren Mengen ein sehr geringer derartiger Niederschlag. erhalten worden; es scheint, dass je nach dem Verhältniss der gemischten Substanzen und der Dauer des Erhitzens die Menge des zurückbleibenden Arsens eine etwas grössere oder geringere, immer aber nur eine sehr unbedeutende ist.

Anders verhält es sich bei der Anwendung von mit Schwefel gemengtem Schwefelarsen; ist die Schwefelmenge irgend bedeutend, so erhält man, wie auch ich mich nochmals überzeugte, gar keinen Spiegel, bei kleineren Schwefelmengen tritt wenigstens eine merkliche Verminderung der Empfindlichkeit ein. So erhielt ich, als ich eine Arsensäurelösung von bekanntem Gehalt mit Schwefelwasserstoff fällte, den Niederschlag mit Ammoniak auszog, wobei etwas freier Schwefel ungelöst blieb und mit der Lösung wie angegeben verfuhr, nur noch aus solchen Quantitäten von Schwefelarsen, die $1 \mathrm{mg}$ und $1 / 10 \mathrm{mg}$ arseniger Säure entsprachen, mit Sicherheit deutliche Spiegel.

Es hat dies jedoch auf das Fresenius-Babo'sche Verfahren gar keinen Bezug, weil bei demselben immer nur das reine (gewogene) Dreifach-Schwefelarsen zur Reduction gelangt und niemals solches, welches aus Arsensäurelösung gefällt ist und deshalb einen höheren Schwefel-

*) Anleitung zur qualitativen Analyse 14. Aufl, s. 386. 
534 Fresenius: Ueber die richtige Ausführung und die Empfindlichkeit

gehalt hat. Da aber bei dem reinen Dreifach-Schwefelarsen durch die Oxydation mit Salpetersäure keine irgend merkliche Zunahme der Genauigkeit erreicht wird, so kann diese Operation ohne Nachtheil unterlassen werden.

3) Die Gasatmosphäre, in welcher die Reduction vorgenommen wird.

R. Fresenius und L. v. Babo schreiben vor, dass die Reduction in einem langsamen Strom trockner Kohlensäure ausgeführt werden soll und diese Angabe ist auch bis in die neueste Zeit in allen Büchern beibehalten worden. Nur an zwei Stellen findet sich ein anderer Vorschlag, nämlich in der fünften Auflage von 0tto's Ausmittelung. der Gifte und in der neuesten Bearbeitung von Graham-0t to's Lehrbuch. Es wird dort nämlich empfohlen statt Kohlensäure Wasserstoff anzuwenden. Robert Otto, von dem diese Abänderung herrührt, gibt zwar an, dass auch Mohr im Wasserstoffstrome reducire, es beruht dies jedoch auf einem Irrthume. Allerdings ist in Mohr's Toxikologie auf Seite 59 ein Apparat abgebildet, der fast vollständig*) mit demjenigen übereinstimmt, den Otto zur Ausführung des Fresenias-Babo'schen Verfahrens empfiehlt.

Bei Mohr soll jedoch der betreffende Apparat keineswegs zur Reduction mittelst Cyankaliums dienen, sondern er ist für das Berzelius'sche Verfahren bestimmt, bei welchem das Schwefelarsen nur mit kohlensaurem Natron gemengt und im Wasserstoffstrome erhitzt wird. Bei dieser Methode muss natürlich Wasserstoff angewendet werden, weil ja sonst gar kein Reductionsmittel vorhanden ist. Bei dem Fres enius-Babo'schen Verfahren gibt $\mathrm{Mohr}$ einen Kohlensäurestrom an und hebt noch besonders die Gründe hervor, die die Verfasser veranlassten, keinen Wasserstoff anzuwenden.**)

Otto glaubt durch seinen Vorschlag eine grössere Empfindlichkeit der Methode zu erreichen, wie sich dies deutlich aus den Worten ergibt, mit denen er ihn begründet:

$\gg$ Fresenius und Babo nehmen die Reduction nicht im Wasser-

*) Otto hat nur statt des bei Mohr abgebildeten Kip p'schen Gasentwicklungsapparates einen solchen empfohlen, wie er in R. Fresenius' Anleiting zur qualitativen Analyse 14. Auf. S. 192 beschrieben ist.

**) Auch schon Rose hat an den angeführten Stellen seines Handbuches auf den Nachtheil des Wasserstoffs hingewiesen. 
»stoffgase, sondern im Kohlensäurestrome vor. Es scheint mir jedoch, »als ob sich das reducirte Arsen aus dem schweren Kohlensäuregase weit »weniger leicht ablagere, als aus dem leichten Wasserstoffgase und des$\gg$ halb empfehle ich in diesem die Reduction auszuführen. Selbst wenn »der Kohlensäurestrom im Momente der Reduction so mässig wie irgend $\gg$ möglich ist, entweicht aus der Reductionsröhre etwas Arsen and erfüllt $\gg$ die Luft mit Knoblauchgeruch, während in einem entsprechenden Wasser»stoffstrome kaum durch den Geruchssinn erkennbare Mengen von Arsen $»$ fortgeführt werden. «

Directe Versuche, aus denen sich eine Erhöhung der Genauigkeit thatsächlich ersehen liesse, hat er nicht ausgeführt, ebenso wenig aber hat er auch berücksichtigt, dass der Anwendung von Wasserstoff ein sehr wichtiges Bedenken*) entgegensteht.

Die Methode würde dadurch nämlich gerade den Vorzug verlieren, welcher von allen Seiten anerkannt und ganz besonders hervorgehoben worden ist, nämlich die Unmöglichkeit einer Verwechslung des Arsens mit Antimon.

Es ergibt sich dies aus den Versuchen, welche R. Fresenius and L. v. Babo in ihrer Originalabhandlung S. 307 und 308 beschrieben haben und die folgendes Resultat lieferten.

Erhitzt man ein Gemenge von Schwefelantimon, Cyankalium und Soda im Kohlensäurestrom, so tritt beim Schmelzen der Masse Reduction ein, es verflüchtigt sich aber nur dann Antimon, resp. es bildet sich nur dann ein Spiegel, wenn dasselbe direct mit dem Gasstrome in Berührung ist. Ist dagegen die Menge des Cyankalium-Sodagemisches hinreichend gross, so bildet dasselbe um jedes Antimonkügelchen eine geschmolzene Schlackenhülle, welche die directe Berührung mit dem Gase, and damit auch die Bildung eines Spiegels verhindert.

Erhitzt man ein ebensolches Gemisch aber im Wasserstoffstrom, so tritt schon vor dem Schmelzen unter dem Einfluss des Wasserstoffes die Reduction ein und die num nicht umhüllten Antimontheilchen verflüchtigen sich in dem Gasstrome.

Es geht hieraus klar hervor, dass man durch Anwendung von

*) Es ist ausserdem nicht zu vergessen, dass es bei weitem weniger leicht gelingt arsenfreie Materialien zur Entwicklung von Wasserstoff als von Kohlensäure zu erhalten. Um einen Arsengehalt des Wasserstoffes zu entfernen Ieitet 0 tto das Gas zuerst durch eine Waschflasche mit ammoniakalischer Silberlösung, wodurch der Apparat immerhin etwas complicirter wird. 
536 Fresenius: Ueber die richtige Ausführung und die Empfindlichkeit etc.

Wasserstoff diesen (auch heute noch) wichtigen Vortheil der Methode aufgeben würde und dass deshalb die Anwendung des Wasserstoffs nicht zulässig ist.

Ich habe die Versuche von R. Fresenius und Babo nochmals wiederholt und ganz die gleichen Resultate erhalten; ich will jedoch nicht unterlassen, darauf aufmerksam zu machen, dass man nur dann auch im Kohlensäurestrom keinen Spiegel erhält, wenn ein hin reichender Ueberschuss ron Cyankalium und Soda vorhanden ist, um eine schützende Hülle um alle Antimontheilchen bilden zu kömnen. Es ist deshalb immer nothwendig, wie dies auch R. Fresenius in der Anleitung zur qualitativen Analyse vorschreibt, sich durch eine vorläufige Prüfung von der $A$ bwesenheit grösserer Antimonmengen zu überzeugen, eventuell dieselben nach dem Meyer'schen Verfahren za entfernen, ehe man zum Reductionsversuche schreitet. Wenn man aber so dafür sorgt, dass nur ganz kleine Mengen von Antimon überhaupt in die Reductionsröhre gelangen können, so ist jede Verwechslung von Arsen und Antimon ausgeschlossen.

Wie schon erwähnt, ist von jeher dieser Umstand als ein ganz besonderer Vorzug der Methode angesehen und von keiner Seite jemals bestritten worden, in neuerer Zeit*) hat man jedoch angefangen, denselben als bedeutungslos anzusehen, da man ja jetzt durch das Mey er'sclie Verfahren in den Stand gesetzt sei, Arsen und Antimon vollständig zu trennen und demnach auch bei dem Verfahren nach Marsh unter keinen Umständen statt eines Arsenspiegels einen Antimonspiegel erhalten könne. Das Meyer'sche Verfahren besteht nun darin, dass man die Sulfide mit salpetersaurem und kohlensaurem Natron schmelzt, sie dadurch in arsensaures und antimonsaures Natron verwandelt, und die Schmelze mit Wasser auszieht. Das Arsen geht dann in Lösung, das Antimon bleibt als antimonsaures Natron im Rückstande. Da jedoch dieses antimonsaure Natron in Wasser nur. schwer löslich und nicht unlöslich ist, so gehen doch immer kleine Mengen von Antimon mit in die Lösung.

Es erschien mir aus diesem Grunde immerhin nicht so ganz unmöglich, dass man, auch bei völliger Abwesenheit von Arsen, aus der wässrigen Lösung im Marsh'schen Apparate doch einen Spiegel erhalten könnte. Und in der That bekam ich, als ich einen derartigen Versuch mit ganz reinem Schwefelantimon ausführte - wobei ich zum Aus-

*) Otto's Ausmittelung der Gifte. 3. Auflage. 
Bericht: Allgemeine analytische Methoden, analyt. Operationen etc. 537

ziehen der Schmelze natürlich nur eine kleine Menge Wasser nahm nachdem ich die wässrige Lösung durch Eindampfen mit Schwefelsäure von Kohlensäure und Salpetersäure befreit hatte, im Marsh'schen Apparate einen zwar kleinen, aber doch sehr deutlichen Antimonspiegel.

Aus dem Vorstehenden geht glaube ich zur Genüge hervor, dass die allein richtige Ausführungsweise der Fresenius-Babo'schen Methode diejenige ist, welche in der ursprünglichen Abhandlung angegeben ist, da die vorgeschlagenen Modificationen sämmtlich keine Verbesserungen der Methode enthalten, sondern im Gegentheil wenigstens theilweise ganz erhebliche Fehler bedingen. Diese Vorschläge konnten, wie ich gezeigt habe, überhaupt nur gemacht werden unter Vernachlässigung der Thatsachen, welche in der Originalabhandlung klar gelegt worden sind. Verfährt man aber in der richtigen Weise, so erhält man nach dieser Methode auch selbst bei nicht sehr grosser Uebung Resultate, welche allen und jeden Anforderungen genügen.

\section{Bericht über die Fortschritte der analytischen Chemie.}

I. Allgemeine analytische Methoden, analytische 0perationen, Apparate und Reagentien.

\section{Von \\ W. Fresenius.}

Eine mikroprismatische Methode zur Unterscheidung fester Substanzen bringt 0 . Maschke*) in Vorschlag. Er geht dabei aus von der Beobachtung, dass kleine Theilchen einer durchsichtigen Substanz, die von einer Flüssigkeit umgeben-sind, welche einen höheren Brechungsexponent hat als der Körper selbst, unter dem Mikroskop sehr charakteristische Färbungen zeigen, deren Intensität mit der Differenz der Brechungsexponenten wächst. Umhüllt man Theilchen einer Substanz, wenn sie auch noch so klein sind, nach und nach mit Flüssigkeiten von verschiedenen Brechungsexponenten, so kamn man verhältnissmässig rasch eine solche finden, die eben erst die leiseste Andeutung dieser Farben hervorruft, also fast genau denselben Brechungsexponenten wie

*) Ann. d. Physik u. Chemie [N. F.] 11, 722. Vom Verfasser eingesandt. 